\title{
Arenavirus Diversity and Evolution: Quasispecies In Vivo
}

\author{
N. Sevilla ${ }^{1}(\bullet) \cdot$ J. C. de la Torre ${ }^{2}$ \\ ${ }^{1}$ Centro de Biología Molecular Severo Ochoa, Universidad Autónoma de Madrid, \\ Campus de Cantoblanco, 28049 Madrid, Spain \\ nsevilla@cbm.uam.es \\ ${ }^{2}$ Department of Neuropharmacology, The Scripps Research Institute, IMM-6 10550 \\ North Torrey Pines Road, La Jolla, CA 92037, USA
}

$1 \quad$ Genetic Variability of Arenaviruses . . . . . . . . . . . . . . 316

1.1 Arenavirus Error-Prone Replication: Mutation, Recombination, and Reassortment . . . . . . . . . . . . . . . . . . . . 317

1.2 Quasispecies Swarms and Adaptive Value of Arenavirus Mutant Spectra . 319

1.3 Mutation Frequencies in Arenavirus Populations . . . . . . . . . . . . . 320

2 Contribution of Arenavirus Genetic Variability to Persistence and Disease . . . . . . . . . . . . . . . . . . 321

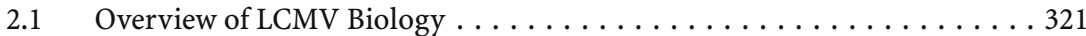

2.2 Selection of LCMV Variants In Vivo: Role in Viral Pathogenesis . . . . . 322

2.2.1 Selection of Immunosuppressive Variants in LCMV Persistently Infected Mice . . . . . . . . . . . . . . . . . . 323

2.2.2 Contribution of LCMV Variants to Virally Induced Growth Hormone Deficiency Syndrome . . . . . . . . . . . . . . . . . . . . 326

$3 \quad$ Perspectives... . . . . . . . . . . . . . . . . . . . . . . . . . . . 329

References . . . . . . . . . . . . . . . . . . . . . . . . . . . 330

Abstract Arenaviruses exist as viral quasispecies due to the high mutation rates of the low-fidelity viral RNA-dependent RNA polymerase (RdRp). This genomic heterogeneity is advantageous to the population, allowing for adaptation to rapidly changing environments that present varying types and degrees of selective pressure. The significant variation in biological properties observed among lymphocytic choriomeningitis virus (LCMV) strains, the prototypic arenavirus, indicates to what extent a quasispecies dynamics may play a role in arenavirus adaptability and pathogenesis. Several aspects of arenavirus variability and its contribution to pathogenesis will be discussed. 
1

\section{Genetic Variability of Arenaviruses}

The family Arenaviridae comprises two distinct complexes: the LCMV-Lassa complex, which includes the Old World arenaviruses, and the Tacaribe virus (TACV) complex, which includes all known New World arenaviruses (Fig. 1). Early sequence analysis of arenaviruses revealed a significant degree of genetic stability with amino acid sequence homologies of $90 \%-95 \%$ among different strains of the prototypic arenavirus lymphocytic choriomeningitis virus (LCMV), whereas significantly higher levels of genetic diversity (37\%$56 \%$ ) were observed for homologous proteins of different arenavirus species (Fulhorst et al. 1996; Southern 1996). More recently, genetic studies with a variety of arenavirus field isolates, including Lassa (Bowen et al. 2000), Junin (Garcia et al. 2000), Guanarito (GTO) (Weaver et al. 2000), Pirital (PIR) (Fulhorst et al. 1999), and Whitewater Arroyo (Fulhorst et al. 2001) have revealed a high degree of genetic variation among geographical and temporal isolates of the same virus species. Notably, a remarkably high level of genetic

\section{Arenaviruses}

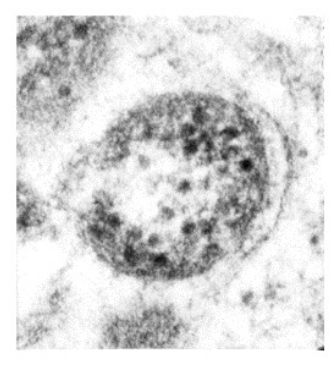

LCMV

Lassa virus
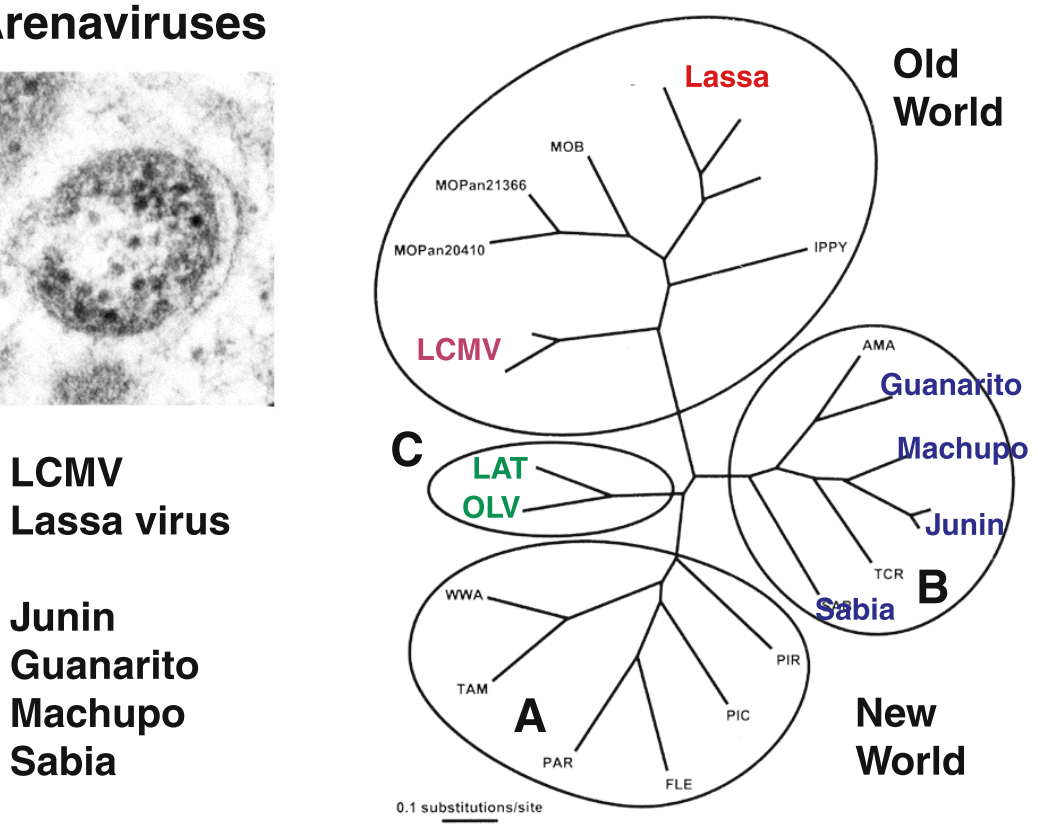

Fig. 1 Phylogenic organization of Arenaviridae and electron micrograph of the prototypic Arenavirus LCMV 
divergence (26\% and $16 \%$ at the nt and amino acid level, respectively) has been documented among PIR isolates within very small geographic regions (Weaver et al. 2001)

The substantial degree of inter- and intra-species genetic variation among arenaviruses appear to have important biological correlates, as suggested by the significant variation in biological properties observed among LCMV strains. In addition, early studies recognized the influence of the passage history on the virus biological properties (Hotchin et al. 1971; Hotchin et al. 1975; Hotchin and Sikora 1973). Thus, early mouse brain passages of LCMV were associated with immunologic tolerance and low mortality in newborn mice. In contrast, late mouse brain passages of LCMV lacked the toleranceinducing capacity and caused high mortality. Likewise, neonatal infection of certain mouse strains with LCMV strains Armstrong (ARM) and E-350 induced growth hormone deficiency syndrome (GHDS) associated with severe hypoglycemia, which frequently resulted in the death of the infected mice. In contrast, infection of the same mouse strains with LCMV strains WE and Traub did not cause GHDS. These striking phenotypic differences were associated with the ability of LCMV ARM and E-350, but not WE and Traub, to replicate at high levels in the GH-producing cells in the anterior pituitary (Oldstone et al. 1985). Moreover, LCMV variants with distinct phenotypes are often selected in different organs of infected mice. Thus, most isolates from the central nervous system (CNS) of mice persistently infected since birth with ARM tend to produce acute infection in adult mice, whereas isolates from the spleen of the same mice exhibit frequently an immunosuppressive phenotype that favors the establishment of persistence (Ahmed and Oldstone 1988). Such dramatic phenotypic differences among genetically closely related LCMV isolates indicate that a few amino acid replacements in LCMV proteins could suffice to produce important alterations in the virus biological properties. This, in turn, raises the question as to what extent quasispecies dynamics may play a role in arenavirus adaptability and pathogenesis.

\section{1}

\section{Arenavirus Error-Prone Replication: Mutation, Recombination, and Reassortment}

The lack of proofreading-repair activities in viral RNA-dependent RNA polymerases (RdRp) is a key determinant of the observed high mutation rates for riboviruses. Thus, mutation rates during RNA genome replication are in the range of $10^{-3}-10^{-5}$ substitutions per nucleotide copied (Drake and Holland 1999; Holland 1992). This means that ribovirus genomes are highly unlikely to undergo two rounds of template copying without introducing an incorrect 
nucleotide into the product. As with other RdRp, the LCMV L polymerase does not contain a recognizable proofreading exonuclease domain. However, it cannot be excluded that the Arenavirus replicase complex could include some cellular subunit with a capacity for error correction.

In addition to mutation, both RNA recombination and genome reassortment can contribute to the genetic variability of arenaviruses. Recombination is particularly relevant for some positive-strand RNA viruses including picornaviruses and coronaviruses. Recombination can facilitate the generation of divergent genome combinations to produce large evolutionary jumps, as well as the rescue of fit genomes from debilitated mutated parents. In contrast, recombination in negative-strand (NS) RNA viruses, including arenaviruses, occurs at frequencies several orders of magnitude lower than in most positivestrand RNA viruses. Moreover, experimental evidence from both laboratory setting experiments and field molecular epidemiology indicates that recombination between two NS ribonucleoproteins (RNPs) is a very rare event, suggesting that perhaps only intramolecular recombination plays a significant role in the evolution of NS RNA viruses. Nevertheless, natural evidence for genetic recombination between two TACV complex members has been reported as the origin whitewater arroyo virus, the most recently discovered arenavirus (Charrel et al. 2001; Fulhorst et al. 1996).

The segmented nature of the arenavirus genome facilitates the generation of reassortant viruses during co-infection of the same cell by two distinguishable parental viruses. In cell culture, reassortant viruses can be generated very efficiently. In some cases, their frequency is close to the theoretical predicted maximum of $50 \%$. However, even among genetically closely related viruses, some particular combinations appear to be excluded. Hence, some specific reassortants are never recovered (Teng et al. 1996a). It is more difficult, however, to assess the contribution of segment reassortment to Arenavirus biology and evolution in the field. As with recombinant viruses, the generation of such reassortants would require co-infection of the same rodent host with two different arenaviruses. Dual infection of the same host may be highly restricted due to homotypic viral interference, which can be readily demonstrated with several arenaviruses including LCMV (Welsh and Pfau 1972) and TV (Gimenez and Compans 1980). This phenomenon is not strictly strain-specific, as illustrated by the existence of interference among different pairs of LCMV strains. Heterotypic interference between arenaviruses has been occasionally reported, and its degree appears to correlate with the genetic relationship of the viruses (Damonte et al. 1983; Welsh and Pfau 1972). This superinfection exclusion phenomenon could contribute to explaining the observed population partitioning in the field, resulting in the maintenance of independent evolutionary lineage of the 
same strain within a small geographic range (Weaver et al. 2001). Nonetheless, it is plausible that in some instances arenavirus reassortants occur and they can display pathogenic potential, which is not manifested by any of the two parental viruses (Riviere 1987; Riviere et al. 1986; Riviere and Oldstone 1986).

\section{2}

\section{Quasispecies Swarms and Adaptive Value of Arenavirus Mutant Spectra}

RNA viruses, even clonal populations, are organized as dynamic mutant distributions termed viral quasispecies (Domingo et al. 2001a; Domingo and Holland 1997). In each infected cell, replicating genomes compete for whatever resources might be limiting (nucleotide substrates, ribosomes, membrane sites, etc.) and needed to complete the virus life cycle. RNAs replicating in two separate cells do not enter into competition and could be regarded as separate quasispecies in separate compartments. However, virus progenies from different cells or tissues may eventually meet in a common compartment (e.g., a new organ such as the brain, or the blood stream), where they may then compete. Error-prone replication might contribute to spatial and temporal heterogeneities in RNA genome populations. Thus for LCMV, selection of organ-specific variants has been extensively documented (Ahmed and Oldstone 1988; Ahmed et al. 1984a).

Viral quasispecies contain a plethora of variants, which allows these quasispecies to display different phenotypes in response to environmental demands. The influence of mutant distributions on the biology of RNA viruses is supported by demonstration of the presence and selection of variants in quasispecies evolving in vivo as well as evidence that mutant spectrum complexity may have a predictive value with regard to the response to antiviral treatments and to shifts from acute into chronic phases of viral disease. The presence of biologically significant variants within quasispecies is well documented by the presence of HIV variants resistant to viral inhibitors in patients who have not been subjected to therapy with the inhibitors, and some of these variants are likely to contribute to viral persistence and pathogenesis (Domingo 2003a, 2003b, Domingo et al. 2001a, 2001b; Domingo and Holland 1997). Although a great majority of mutations harbored in mutant swarms of RNA virus populations may not have either short- or long-term selective value, the evolution of viral quasispecies can, however, be adaptive and influence viral pathogenesis (Domingo 2001a, 2003a; Domingo and Holland 1997).

Increasing evidence indicates that the complexity of mutant spectra influences the virus pathogenic potential, response to treatments and its ability to establish chronicity (Domingo 2003a, 2003b; Domingo et al. 2001b). Moreover, 
it seems plausible that parameters that can be quantified in viral quasispecies could be used as surrogate markers to anticipate the virus responses to treatment and its potential for chronicity (Farci et al. 2000; Pawlotsky et al. 1998).

\section{3}

\section{Mutation Frequencies in Arenavirus Populations}

No systematic measurements of mutant spectrum complexity have been reported for arenavirus populations. However, CTL escape mutants were readily selected in T cell receptor (TCR) transgenic mice infected with high doses $\left(10^{6} \mathrm{PFU}\right)$ of LCMV (Pircher et al. 1990). Escape mutants included single amino acid replacements in T cell epitopes. CTLs from infected, transgenic mice lysed target cells coated with peptides representing the wild-type $T$ cell epitopes, but not cells coated with variant peptides. Observations in cell culture also suggest high frequencies of CTL escape mutants. The latter were readily selected when B6-SV40 primary fibroblasts were infected, under CTL selective pressure, at a multiplicity of infection (MOI) of 1 or $10^{-3}$, but not $10^{-5}$, suggesting that escape mutants either pre-existed in the LCMV population or occurred early in the course of one replication round in the infected cells (Aebischer et al. 1991). Although CTLs play a key role in the control of LCMV infection, a neutralizing antibody response may be involved in the control of viremia and viral clearance in the absence of $\mathrm{CD} 8^{+}$ $T$ cells (Ciurea et al. 2001). High doses of LCMV-WE led to an enhanced antibody response, and control of virus replication in $\mathrm{CD} 8^{-/-}$mice early on during infection. Nonetheless, neutralizing-resistant LCMV mutants emerged after weeks of CTL absence. The mutants included amino acid substitutions within GP1, and manifested a strong tendency to persist in mice. In addition to amino acid replacements, silent mutations (synonymous, not leading to amino acid substitutions) were found at frequencies of $3 \times 10^{-4}$ substitutions per nucleotide, which would argue for considerable complexity of LCMV in these infected mice.

Recent studies exploring the use of lethal mutagenesis to combat arenavirus infections have provided more direct estimates of the error frequency associated with RNA synthesis mediated by the Arenavirus polymerase (GrandePerez et al. 2002; Ruiz-Jarabo et al. 2003). The observed mutation frequencies of $2.6 \times 10^{-4}$ to $5.5 \times 10^{-4}$ for the NP, GP, and L regions analyzed are consistent with previous findings on genetic heterogeneity of LCMV (Sevilla et al. 2002) and other arenaviruses (Bowen et al. 1996, 1997, 2000; Charrel et al. 2003; Fulhorst et al. 2001; Weaver et al. 2000, 2001), and also within the range commonly observed for riboviruses (Domingo et al. 2001a). 


\section{Contribution of Arenavirus Genetic Variability to Persistence and Disease}

\section{1 \\ Overview of LCMV Biology}

The prototypic Arenavirus LCMV has been used extensively as a model system for the study of viral persistence and pathogenesis. LCMV can infect its natural host, the mouse, either acutely or persistently. The outcome of the infection depends on both host and viral determinants, including the genetics, age, and immune status of the host, as well as route and dose of the virus inoculum (Buchmeier et al. 2001; Buchmeier and Zajac 1999). Investigations using this viral model have been central to defining basic virologic and immunologic concepts including the major histocompatibility complex (MHC) restriction of $\mathrm{T}$ cell recognition, tolerance, immunological memory, immune-mediated pathology, as well as the strategies by which viruses evade the host immune responses (Buchmeier et al. 2001; Buchmeier and Zajac 1999). Studies of LCMV virus-host interaction have also uncovered the ability of noncytolytic persistent viruses to induce disease by disrupting specialized functions of infected cells, revealing a new way by which viruses do harm in the absence of the classic hallmarks of cytolysis and inflammation (de la Torre and Oldstone 1996, 1989, 1991, 1998, 2002; Oldstone et al. 1982).

LCMV is an enveloped virus with a bisegmented negative strand (NS) RNA genome (Buchmeier et al. 2001; Meyer et al. 2002) (Fig. 2). Each of the twogenome segments, designated $\mathrm{L}(\sim 7.2 \mathrm{~kb})$ and $\mathrm{S}(\sim 3.4 \mathrm{~kb})$, expresses two viral gene products using an ambisense coding strategy. The $S$ RNA directs the synthesis of the nucleoprotein, NP, (ca $63 \mathrm{kDa}$ ) and the GP-C enveloped glycoprotein precursor. NP, encoded in antigenome polarity, is the most abundant viral protein and encapsidates viral genomes and antigenomic replicative intermediates. GP-C, encoded in genome polarity, is post-translationally cleaved by the cellular subtilase S1P into mature viral glycoproteins, GP-1 (40-46 kDa) and GP-2 (35 kDa) (Beyer et al. 2003; Pinschewer et al. 2003). Noncovalently associated GP1/GP2 complexes make up the spikes on the virion envelope, and mediate virus interaction with the host cell receptor (Cao et al. 1998; Kunz et al. 2002). The L segment codes for the virus RNA-dependent RNA polymerase (RdRp) (L, 200 kDa) (Salvato et al. 1989) and a small (11 kDa) RING finger protein called Z (Salvato 1993; Salvato et al. 1992; Salvato and Shimomaye 1989), which functions as the arenavirus counterpart of the matrix protein found in many NS RNA viruses (Perez et al. 2003). Additional roles of $\mathrm{Z}$ in the Arenavirus life cycle have been proposed based on its interaction with several host cell proteins and its ability to inhibit RNA synthesis mediated 


\section{Lymphocytic choriomeningitis virus (LCMV)}

\section{Virion Structure}

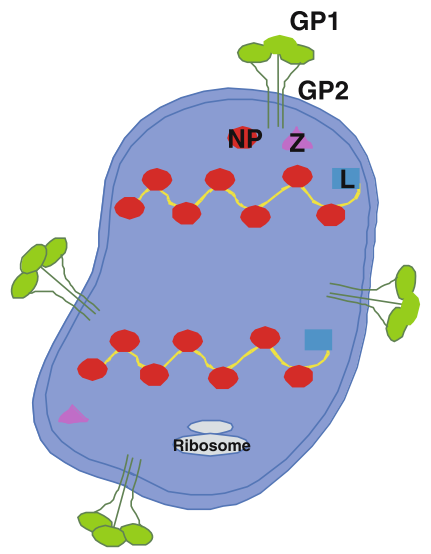

Genome Organization

S RNA (3.5 kb)

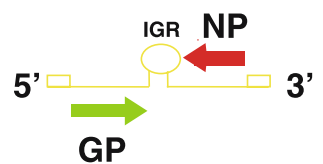

L RNA (7.2 kb)

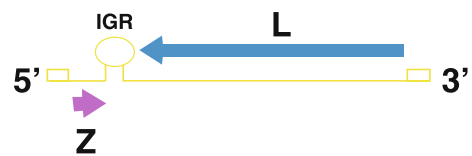

Fig. 2 Schematic representation of the Arenavirus genome organization and virion structure

by the LCMV polymerase (Borden et al. 1998a, 1998b, Campbell Dwyer et al. 2000; Cornu and de la Torre 2001, 2002).

Closely related variants of the same LCMV strain can display remarkable phenotypic differences in vivo, including those related to disease manifestations. We will next discuss two different LCMV-host interactions that illustrate how the generation and selection of specific variants during the natural course of LCMV infection can significantly influence the outcome of the infection.

\section{2}

\section{Selection of LCMV Variants In Vivo: Role in Viral Pathogenesis}

When immunocompetent adult mice are injected with LCMV, they generate a marked immune response to eliminate the infectious agent. Virus clearance is mediated by major histocompatibility complex (MHC) class-I restricted $\mathrm{CD}^{+}$antiviral cytotoxic T lymphocytes (CTL) (Borrow and Oldstone 1997; Buchmeier and Zajac 1999; Oldstone 2002). In contrast, mice infected neonatally or in uterus with LCMV become persistently infected for life. This persistent infection is due to the infection of thymic cells and the specific removal (negative selection) of lymphocytes with potential responsiveness to LCMV (Borrow and Oldstone 1997; Buchmeier and Zajac 1999; Oldstone 2002). This 
long-term persistent infection allows the generation of viral variants that have specific growth advantages in certain tissues and can display phenotypic differences in vivo, including those related to disease manifestations.

Several members of the arenavirus family use $\alpha$-dystroglycan ( $\alpha$-DG) as a cellular receptor, including the Old World arenaviruses LCMV, Lassa fever virus (LFV), and Mobala as well as Clade C New World arenaviruses (Kunz et al. 2002). This receptor, $\alpha-D G$, is a ubiquitously expressed, highly versatile cell surface receptor that provides a molecular link between the extracellular matrix (ECM) and the actin-based cytoskeleton and plays a critical role in cell-mediated assembly of basement membranes. Differences in binding properties to $\alpha$-DG have been documented among arenaviruses, and such differences have been linked to differences in virus-host interactions, including tropism and pathogenesis, which is discussed below.

\subsection{1}

\section{Selection of Immunosuppressive Variants in LCMV Persistently Infected Mice}

In the course of persistent infections of mice with LCMV, distinct viral variants can be isolated from the brain and lymphoid tissue (Ahmed et al. 1984b). These viral variants have different biological properties that correlate with the type of tissue from which they are isolated. One type of variant predominates in the CNS (Evans et al. 1994), whereas the other type predominates in lymphocytes and macrophages of the immune system (Fig. 3) (Ahmed and Oldstone 1988; Ahmed et al. 1984a). Most of the CNS isolates are similar to the parental Armstrong (Arm) strain used to infect the mice and induce a potent virusspecific CTL responses in adult mice, in which the infection is cleared within 2 weeks. In contrast, the majority of the isolates derived from the lymphoid tissue cause chronic infections in adult mice associated with absence of LCMVspecific CTL response, absence of CTL response to other viruses, and absence to antibody responses to soluble and foreign antigens (Ahmed and Oldstone 1988; Ahmed et al. 1984a). The prototypic member of the immunosuppressive variants is clone $13(\mathrm{Cl} 13)$, which was derived from the spleen of a mouse persistently infected from birth with the nonsuppressive LCMV Arm isolate. Sequence analysis revealed only two amino acid changes in $\mathrm{Cl} 13$ compared with the parental Arm, F260L in the viral glycoprotein-1 (GP1) (Salvato et al. 1988), and K1079Q in the viral polymerase encoded by the L gene (Salvato et al. 1991). Injection of mice with reassortant viruses between $\mathrm{Cl} 13$ and Arm showed that only reassortants containing the S segment (GP1) that came from Arm exhibited high levels of LCMV-specific CTL and the infection was cleared in 2 weeks (Matloubian et al. 1993). These findings indicate that the immunosuppressive potential of $\mathrm{Cl} 13$ maps to the $\mathrm{S}$ segment, with strong 
evidence for an association of amino acid substitution F260L in GP1 with the immunosuppressive phenotype (Fig. 3). The emergence of cell-specific viral variants can be explained by the fact that when a viral infection occurs in the whole animal, the various organs and cell types present in the body provide a rich milieu for the selection of viral variants. During long-term persistence in carrier mice with continuous virus replication, and given the high mutation rate of RNA viruses, LCMV variants that have a growth advantage in certain cell types are likely to emerge. In addition, the contribution of the selective

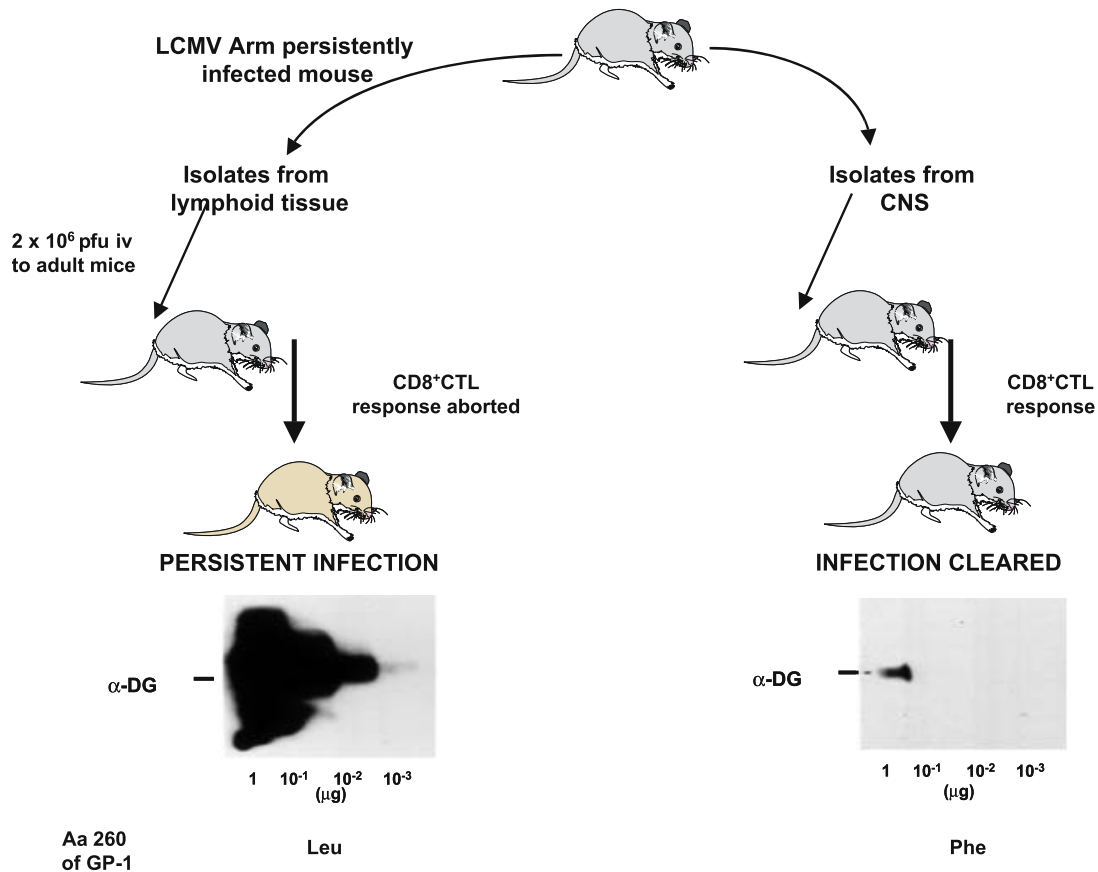

Fig. 3 Selection of viral variants from mice persistently infected at birth with LCMV Arm. Variants isolated from a variety of immune cells (lymphoid tissue), when inoculated intravenously into adult immunocompetent mice, fails to generate a sufficient primary day-7 CTL response to clear viral infection resulting in a persistent viral infection (immunosuppressive variants). In contrast, those variants isolated from the CNS, upon inoculation of adult immunocompetent mice, generate a robust CTL response and clear the virus infection (nonimmunosuppressive). The immunosuppressive variants bind at high affinity to $\alpha$-DG immobilized on membranes while the nonimmunosuppressive variants bind at low affinity to $\alpha$-DG. The difference in GP amino acid residues is a single amino acid at position 260 , having a Leu the immunosuppressive variants as opposed to the nonimmunosuppressive with a Phe at that site 
pressure of the host immune system has to be taken into consideration. In order to evaluate all these components, the selection of a large number of LCMV variants in vivo has been documented (Sevilla et al. 2000). The vast majority of viruses isolated from lymphocytes and macrophages of CD4 knock out (ko), perforin ko, and TNF- $\alpha$ ko mice persistently infected with Arm showed an immunosuppressive phenotype when inoculated i.v. into adult immunocompetent mice. In contrast, all the CNS viral isolates from the same mice exhibited a nonimmunosuppressive phenotype. Selection of immunosuppressive and nonimmunosuppressive variants occurred at similar frequencies in normal and immunocompromised mice, indicating that it is highly unlikely that the host immune response represents a main driving force in this selection process. Sequence analysis of a large number of these variants consistently showed an amino acid exchange F260L or F260I in GP1 of the immunosuppressive viruses. As the selection of such viral variants is influenced in a critical way by the host tissue, it may involve, among other factors, virus-receptor interactions (Sevilla et al. 2000). In fact, these viruses showed a 200- to 500-fold enhanced binding to $\alpha-D G$ and a high dependence on $\alpha$-DG for the infection of cells (Kunz et al. 2002). The analysis of the anatomic distribution of immunosuppressive variants in the spleen of adult immunocompetent mice 3 days after infection showed a different tropism for both group of variants (Fig. 4) (Sevilla et al. 2000). Cl13-like viruses localized exclusively in cells of the marginal zone and white pulp of spleen, whereas Arm-like viruses localized primarily in cells within the red pulp. This distinct distribution in spleen indicated that different subsets of cells were infected by the immunosuppressive and the nonimmunosuppressive viruses. The identification of cells in the spleen infected by LCMV revealed two subsets mainly infected by immunosuppressive viruses, CD11c- and DEC-205 positive dendritic cells (Fig. 4) (Sevilla et al. 2000). The major cell population expressing $\alpha$-DG in spleen is $\mathrm{CD} 11 \mathrm{c}^{+}$cells. All these data together suggest that those viral variants with increased binding receptor affinity might be selected within the replicating quasispecies to bind to $\mathrm{CD} 11 \mathrm{c}^{+}$and DEC- $205^{+}$ cells. The selective pressure is likely based on the interaction of the virus in initiating the dysfunction of the host antigen presentation, aborting the necessary host immune responses required to terminate the viral infection (Sevilla et al. 2004).

The differential tropism of immunosuppressive (high-affinity binders) and nonimmunosuppressive (low-affinity binders) LCMV variants may relate to their ability to compete with host-derived ligands of $\alpha$-DG. Variants with high-affinity receptor binding may out-compete host-derived ligands of $\alpha$ DG, such as Arm, and may be blocked by host-derived ligands. Given the highly complex tissue-specific binding pattern of dystroglycan, the ability of 
LCMV immunosuppressive

a variants

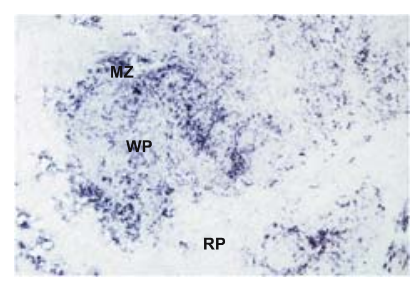

b

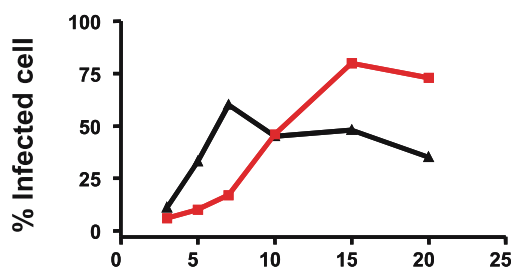

LCMV non-immunosuppressive variants

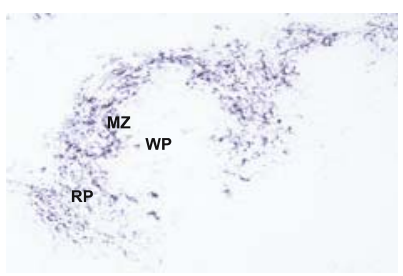

Days post-infection

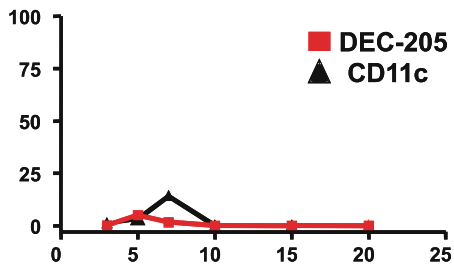

Fig. 4a,b Differential tropism of LCMV immunosuppressive and nonimmunosuppresive variants in vivo. a Viral nucleic acid sequences of these variants located the immunosuppressive to the white pulp of spleen and the nonimmunosuppressive to the red pulp. b Replication of immunosuppressive variants in CD11 $\mathrm{c}^{+}$and DEC-205 dendritic cells (up to $80 \%$ of such cells are infected) is shown. By comparison, $<12 \%$ of such cells are infected by LCMV nonimmunosuppressive variants

a viral variant to compete for dystroglycan binding with host-derived ligands may be crucial for the infection of a particular cell type such as dendritic cells. Both in vivo and in vitro competition assays between Arm and $\mathrm{Cl} 13$ showed that Arm dominates over $\mathrm{Cl} 13$ in neurons, but $\mathrm{Cl} 13$ has a higher fitness than Arm during replication in liver and spleen (Evans et al. 1994; King et al. 1992). This illustrates the relationship between virus-host molecular interactions and the selection of biologically relevant viral variants.

\subsection{2}

\section{Contribution of LCMV Variants to Virally Induced Growth Hormone Deficiency} Syndrome

LCMV induced growth hormone (GH) deficiency syndrome (GHDS) is manifested by marked growth retardation and the development of severe hypoglycemia, which frequently leads to the death of the infected mice (Oldstone 
et al. 1982, 1984). Both viral and host genetic determinants contribute to GHDS (Oldstone 2002; Oldstone et al. 1982, 1984, 1985; Riviere et al. 1985a, Tishon and Oldstone 1990). The development of GHDS is associated with high viral load in the GH-producing cells of the anterior pituitary, despite an absence of noticeable tissue structural damage or inflammation, yet levels of GH, both mRNA and protein are significantly diminished (Klavinskis and Oldstone 1986, 1989; Oldstone et al. 1985; Valsamakis et al. 1987). Studies using a pituitary-derived somatotroph cell line, PC, indicated that LCMVinduced reduction in GH synthesis is likely due to the altered activity of the GH transcriptional transactivator GHF1 (Pit-1) in the infected cells (de la Torre and Oldstone 1992, 1996). This, in turn, results in lower levels of GH promoter activity and the subsequent decrease in mRNA synthesis and protein production.

Studies using reassortant viruses between strains of LCMV, which do (ARM), or do not (WE) cause GHSD, mapped the ability to cause this disorder to the S RNA (Riviere et al. 1985b). As predicted, clonal analysis of the parental GHDS-nil WE population showed that the majority (58/61) of the clones examined behaved as the parental WE clonal population and did not cause GHDS (Buesa-Gomez et al. 1996). Notably, however, three of the 61 clones isolated from the WE parental population did cause the characteristic GHDS (Buesa-Gomez et al. 1996). These results provided evidence that variants with the ability to cause GHDS $\left(\mathrm{GHDS}^{+}\right.$) may constitute up to $5 \%$ of the RNA species within a GHDS-nil replicating WE parental population. WE variants that caused GHDS replicated at high levels in the GH-producing cells of the anterior pituitary and caused reduced levels of GH synthesis in the infected mice. In contrast, the majority of the WE clonal isolates had the same GHDS-nil phenotype as the parental WE and replicated poorly in the GH-producing cells (Buesa-Gomez et al. 1996).

Clonal isolates from the same parental virus population are expected to be genetically very closely related. Therefore, the molecular characterization of GHDS-nil (e.g., Wec54) and GHDS ${ }^{+}$(e.g., Wec2.5) WE variants facilitated the investigation of the precise viral determinants required for the development of GHDS. The biological characterization of reassortants between WEc54 or WEc2.5 variants and ARM $\left(\mathrm{GHDS}^{+}\right)$, confirmed that the virus's ability to cause GHDS was associated with the S RNA (Teng et al. 1996a). Sequence analysis of the S RNA of WEc54 and WEc2.5 showed that the genetic basis for their phenotypic differences is a single amino acid substitution $\mathrm{S}$ to $\mathrm{F}$ at position 153 in the GP1 (Teng et al. 1996a). With the exception of their different tropism for GH-producing cells, both WEc54 and WEc2.5 have the same tissue and cell distribution in GHDS susceptible infected mice. Therefore, the single amino acid substitution S153F in GP-1 appears to confer WEc2.5-like variants with 
the ability to recognize a virus receptor that is specifically expressed in the GH-producing cells. PC cells did not exhibit the restriction for LCMV entry observed in vivo. Both GHDS (+) and (-) LCMV variants were able to replicate in PC cells and both caused a similar decrease in GH mRNA steady-state levels (Teng et al. 1996a). These findings suggest that in addition to GP-1, involved in receptor recognition and virus entry into the GH target cells, other viral determinants also contribute to LCMV-mediated impairment of GH synthesis.

Interestingly, WEc54 and WEc2.5 also exhibited distinct $\mathrm{CTL}^{-} \mathrm{P}^{+}$and $\mathrm{CTL}^{+} \mathrm{P}^{-}$phenotypes, respectively. As with $\mathrm{Cl} 13$; The $\mathrm{CTL}^{-} \mathrm{P}^{+}$phenotype displayed by WEc 54 correlated with the virus's ability to target dendritic cells in the spleen. It is then intriguing that the same mutation S153F in GP-1 extends the tropism of WE to the GH-producing cells, but abrogates its capability to target dendritic cells. The mechanisms underlying the association of a single amino acid at position 153 in GP-1 with two different and seemingly unrelated phenotypes, namely, the ability to cause GHDS and immunosuppression, remain to be determined. Nevertheless, these findings illustrate how only minor changes in the virus genome may have a great impact in the biology of the infected host.

An intriguing question is how $\mathrm{GHDS}^{+} \mathrm{WE}$ variants are stably maintained, although phenotypically silent, within the GHDS-nil WE population. Likewise, it would be important to determine whether changes in the virus population dynamics within the infected host influence disease progression. The single S153F substitution in GP1 associated with the phenotypic difference between WEc54 (GHDS-nil) and Wec2.5 (GHDS+) was caused by a single nucleotide substitution $\mathrm{G}$ to $\mathrm{A}$. As a consequence of this nucleotide change, cDNAs derived from WEc54 and WEc2.5 differ by the presence or absence, respectively, of a EcoRI site, which provided the basis for a sensitive RT-PCR and restriction enzyme cleavage assay to analyze the dynamics of these WE variants during the natural course of infection (Teng et al. 1996b). Results from these studies indicated that neonatal $\mathrm{C} 3 \mathrm{H} / \mathrm{St}$ mice infected with a mixture of Wec54 (GHDS-nil) and Wec2.5 (GHDS ${ }^{+}$) developed GHDS only when Wec2.5 represented a fraction higher than 0.1 in an inoculum of 1,000 PFU (Teng et al. 1996b). Importantly, mice infected with less than $10 \mathrm{PFU}$ of Wec2.5 developed GHDS, indicating that the amount of WEc2.5 (10 PFU), corresponding to a fraction of 0.1 in the inoculum of the mix virus population, should have been sufficient to induce GHDS. Interestingly, WEc2.5 was not entirely out-competed by a large excess of Wec 54 and was stably maintained at low levels, but phenotypically silent, in the infected mouse. As predicted, Wec54 and WEc2.5 also exhibited quasispecies structures but with sequence distributions that differed between them and with respect to the parental WE population (Teng et al. 1996b). About 50\% (14/32) of the analyzed clones 
derived from the EcoRI-resistant PCR band obtained from the RNA of cells infected with the parental WE population had the G to A change resulting in the S153F GP-1 mutation present in Wec2.5. In contrast, when the same analysis was applied to the WEc54 quasispecies, none of the 28 clones analyzed had the WEc2.5 genotype (Teng et al. 1996b). These findings illustrate how differences in the sequence distributions between two quasispecies with very similar, or identical, consensus sequences can significantly influence the virus's impact on the infected host.

Direct fitness measurements of WEc54 and WEc2.5 variants have not been performed. However, one-step growth studies in cultured cells showed that WEc2.5 appears to replicate slightly faster and to higher titers than WEc54. The question then is why WEc2.5 species do not raise to dominance over WEc54 species within the WE population. In this respect, studies with VSV have shown that virus clones with relatively high fitness could be hidden and suppressed by viral quasispecies containing a large excess of variants with lower average fitness (de la Torre and Holland 1990). These findings would suggest that the phenotypic manifestations, including disease symptoms, of a virus infection can be significantly influenced by the levels at which particular variants are present within the population. Hence, pathogenic variants, including those having high fitness, can be maintained at low levels, and thereby phenotypically silent, within a nonpathogenic viral population. Upon bottleneck transmission events, levels of a previously phenotypically suppressed variant might reach the required threshold for its phenotypic manifestation, which can drastically alter the outcome of the infection in the newly infected host or tissue.

\section{3}

\section{Perspectives}

It is now well established that most, if not all, RNA viruses have the genetic structure of quasispecies. Increasing amounts of information obtained from different viral systems are contributing to the elucidation of the complex dynamics of viral quasispecies. This is important to understand not only virus evolution, but also viral pathogenesis. Such population dynamics may often confound efforts to correlate phenotypic manifestations of virus-induced disease with single genome variants of a defined sequence. Viral systems, like LCMV, for which both cell culture systems and suitable small laboratory animal models are available, provide us with ideal tools for the investigation of the impact of viral quasispecies on the biology of the infected host. The findings on LCMV-host interactions we have discussed here would support 
the view that persistent viral infections can be viewed as a succession of invasions modulated by the response of the host. The manifestations of these chronic infections will differ not only because of the genetic, physiological and immunological differences among the infected hosts, but also because each host is likely to face a unique array of quasispecies challenges during infection. These features of viral infections are mostly overlooked because disease symptoms are often, but not always, similar for a given type of virus. It is worth noting that variants, present within a given viral quasispecies, and with the potential of exerting a very different impact on the biology of the infected host can, however, exhibit indistinguishable biochemical and immunological properties. Hence, the use of classic biochemical and genetic assays that define features of the consensus population sequence may fail to detect the presence of variants capable of triggering different disease symptoms. This, in turn, might lead clinicians to disregard the contribution of relevant infectious agents to certain disease manifestations.

\section{References}

Aebischer T, Moskophidis D, Rohrer UH, Zinkernagel RM, Hengartner H (1991) In vitro selection of lymphocytic choriomeningitis virus escape mutants by cytotoxic T lymphocytes. Proc Natl Acad Sci U S A 88:11047-11051

Ahmed R, Oldstone MB (1988) Organ-specific selection of viral variants during chronic infection. J Exp Med 167:1719-1724

Ahmed R, Salmi A, Butler LD, Chiller JM, Oldstone MB (1984a) Selection of genetic variants of lymphocytic choriomeningitis virus in spleens of persistently infected mice. Role in suppression of cytotoxic $\mathrm{T}$ lymphocyte response and viral persistence. J Exp Med 160:521-540

Ahmed R, Salmi A, Butler LD, Chiller JM, Oldstone MBA (1984b) Selection of genetic variants of lymphocytic choriomeningitis virus in spleens of persistently infected mice: role in suppression of cytotoxic $\mathrm{T}$ lymphocyte response and viral persistence. J Exp Med 160:521-540

Beyer WR, Popplau D, Garten W, von Laer D, Lenz O (2003) Endoproteolytic processing of the lymphocytic choriomeningitis virus glycoprotein by the subtilase SKI1/S1PJ Virol 77:2866-2872

Borden KL, Campbell Dwyer EJ, Salvato MS (1998a) An arenavirus RING (zincbinding) protein binds the oncoprotein promyelocyte leukemia protein (PML) and relocates PML nuclear bodies to the cytoplasm. J Virol 72:758-766

Borden KL, Campbelldwyer EJ, Carlile GW, Djavani M, Salvato MS (1998b) Two RING finger proteins, the oncoprotein PML and the arenavirus $\mathrm{Z}$ protein, colocalize with the nuclear fraction of the ribosomal P proteins. J Virol 72:3819-3826

Borrow P, Oldstone MBA (1997) Lymphocytic choriomeningitis virus. In: Nathanson $\mathrm{N}$ et al. (eds) Viral pathogenesis, Lippincott-Raven, Philadelphia, pp 593-619

Bowen MD, Peters CJ, Nichol ST (1996) The phylogeny of New World (Tacaribe complex) arenaviruses. Virology 219:285-290 
Bowen MD, Peters CJ, Nichol ST (1997) Phylogenetic analysis of the Arenaviridae: patterns of virus evolution and evidence for cospeciation between arenaviruses and their rodent hosts. Mol Phylogenet Evol 8:301-316

Bowen MD, Rollin PE, Ksiazek TG, Hustad HL, Bausch DG, Demby AH, Bajani MD, Peters CJ, Nichol ST (2000) Genetic diversity among Lassa virus strains. J Virol 74:6992-7004

Buchmeier MJ, Zajac AJ (1999) Lymphocytic choriomeningitis virus. In: Chen J, Ahmed R (eds) Persistent viral infections, 1st edn. John Wiley, Boston, pp 575-605

Buchmeier MJ, Bowen MD, Peters CJ (2001) Arenaviridae: the viruses and their replication. In: Knipe DM, Howley PM (eds) Fields virology, 4th edn. Lippincott Williams, Wilkins, Philadelphia, pp 1635-1668

Buesa-Gomez J, Teng MN, Oldstone CE, Oldstone MB, de la Torre JC (1996) Variants able to cause grow th hormone deficiency syndrome are present within the diseasenil WE strain of lymphocytic choriomeningitis virus. J Virol 70:8988-8992

Campbell Dwyer EJ, Lai H, MacDonald RC, Salvato MS, Borden KL (2000) The lymphocytic choriomeningitis virus RING protein $\mathrm{Z}$ associates with eukaryotic initiation factor $4 \mathrm{E}$ and selectively represses translation in a RING-dependent manner. J Virol 74:3293-3300

Cao W, Henry MD, Borrow P, Yamada H, Elder JH, Ravkov EV, Nichol ST, Compans RW, Campbell KP, Oldstone MB (1998) Identification of alpha-dystroglycan as a receptor for lymphocytic choriomeningitis virus and Lassa fever virus. Science 282:2079-2081

Charrel RN, de Lamballerie X, Fulhorst CF (2001) The Whitewater Arroyo virus: natural evidence for genetic recombination among Tacaribe serocomplex viruses (family Arenaviridae) Virology 283:161-166

Charrel RN, Lemasson JJ, Garbutt M, Khelifa R, De Micco P, Feldmann H, de Lamballerie X (2003) New insights into the evolutionary relationships between arenaviruses provided by comparative analysis of small and large segment sequences. Virology 317:191-196

Ciurea A, Hunziker L, Zinkernagel RM, Hengartner H (2001) Viral escape from the neutralizing antibody response: the lymphocytic choriomeningitis virus model. Immunogenetics 53:185-189

Cornu TI, de la Torre JC (2001) RING finger Z protein of lymphocytic choriomeningitis virus (LCMV) inhibits transcription and RNA replication of an LCMVS-segment minigenome. J Virol 75:9415-9426

Cornu TI, de la Torre JC (2002) Characterization of the arenavirus RING finger Z protein regions required for Z-mediated inhibition of viral RNA synthesis. J Virol 76:6678-6688

Damonte EB, Mersich SE, Coto CE (1983) Response of cells persistently infected with arenaviruses to superinfection with homotypic and heterotypic viruses. Virology 129:474-478

De la Torre JC, Holland JJ (1990) RNA virus quasispecies populations can suppress vastly superior mutant progeny. J Virol 64:6278-6281

De la Torre JC, Oldstone MB (1992) Selective disruption of growth hormone transcription machinery by viral infection. Proc Natl Acad Sci U S A 89:9939-9943

De la Torre JC, Oldstone MBA (1996) The anatomy of viral persistence: Mechanisms of persistence and associated disease. Adv Virus Res 46:311-343 
Domingo E (2003a) Host-microbe interactions: viruses. Complexities of virus-cell interactions. Curr Opin Microbiol 6:383-385

Domingo E (2003b) Quasispecies and the development of new antiviral strategies. Prog Drug Res 60:133-158

Domingo E, Holland JJ (1997) RNA virus mutations and fitness for survival. Annu Rev Microbiol 51:151-178

Domingo E, Biebricher C, Eigen M, Holland JJ (2001a) Quasispecies and RNA virus evolution: principles and consequences. Austin, Landes Bioscience

Domingo E, Mas A, Yuste E, Pariente N, Sierra S, Gutierrez-Riva M, Menendez-Arias L (2001b) Virus population dynamics, fitness variations and the control of viral disease: an update. Prog Drug Res 57:77-115

Drake JW, Holland JJ (1999) Mutation rates among RNA viruses. Proc Natl Acad Sci U S A 96:13910-13913

Evans CF, Borrow P, de la Torre JC, Oldstone MB (1994) Virus-induced immunosuppression: kinetic analysis of the selection of a mutation associated with viral persistence. J Virol 68:7367-7373

Farci P, Shimoda A, Coiana A, Diaz G, Peddis G, Melpolder J C, Strazzera A, Chien DY, Munoz SJ, Balestrieri A, Purcell RH, Alter HJ (2000) The outcome of acute hepatitis C predicted by the evolution of the viral quasispecies. Science 288:339-344

Fulhorst CF, Bowen MD, Ksiazek TG, Rollin PE, Nichol ST, Kosoy MY, Peters CJ (1996) Isolation and characterization of Whitewater Arroyo virus, a novel North American arenavirus. Virology 224:114-120

Fulhorst CF, Bowen MD, Salas RA, Duno G, Utrera A, Ksiazek TG, De Manzione NM, De Miller E, Vasquez C, Peters CJ, Tesh RB (1999) Natural rodent host associations of Guanarito and pirital viruses (Family Arenaviridae) in central Venezuela. Am J Trop Med Hyg 61:325-330

Fulhorst CF, Charrel RN, Weaver SC, Ksiazek TG, Bradley RD, Milazzo ML, Tesh RB, Bowen MD (2001) Geographic distribution and genetic diversity of Whitewater Arroyo virus in the southwestern United States. Emerg Infect Dis 7:403-407

Garcia JB, Morzunov SP, Levis S, Rowe J, Calderon G, Enria D, Sabattini M, Buchmeier MJ, Bowen MD, St Jeor SC (2000) Genetic diversity of the Junin virus in Argentina: geographic and temporal patterns. Virology 272:127-136

Gimenez HB, Compans RW (1980) Defective interfering Tacaribe virus and persistently infected cells. Virology 107:229-239

Grande-Perez A, Sierra S, Castro MG, Domingo E, Lowenstein PR (2002) Molecular indetermination in the transition to error catastrophe: systematic elimination of lymphocytic choriomeningitis virus through mutagenesis does not correlate linearly with large increases in mutant spectrum complexity. Proc Natl Acad Sci U S A 99:12938-12943

Holland JJ (1992) Genetic diversity of RNA viruses. CTM Immunol (ed) SpringerVerlag, Berlin Heidelberg New York

Hotchin J, Sikora E (1973) Low-pathogenicity variant of lymphocytic choriomeningitis virus. Infect Immun 7:825-826

Hotchin J, Kinch W, Benson L (1971) Lytic and turbid plaque-type mutants of lymphocytic choriomeningitis virus as a cause of neurological disease or persistent infection. Infect Immun 4:281-286 
Hotchin J, Kinch W, Benson L, Sikora E (1975) Role of substrains in persistent lymphocytic choriomeningitis virus infection. Bull World Health Organ 52:457-463

King CC, Jamieson BD, Reddy K, Bali N, Concepcion RJ, Ahmed R (1992) Viral infection of the thymus. J Virol 66:3155-3160

Klavinskis LS, Oldstone MB (1986) Perturbation of endocrine functions during persistent infection of mice with lymphocytic choriomeningitis virus. Med Microbiol Immunol (Berl) 175:117-119

Klavinskis LS, Oldstone MB (1989) Lymphocytic choriomeningitis virus selectively alters differentiated but not housekeeping functions: block in expression of growth hormone gene is at the level of transcriptional initiation. Virology 168:232-235

Kunz S, Borrow P, Oldstone MB (2002) Receptor structure binding and cell entry of arenaviruses. In: Oldstone MB (ed) Arenaviruses. Curr Topics Microbiol Immunol 267:111-138

Matloubian M, Kolhekar SR, Somasundarum T, Ahmed R (1993) Molecular determinants of macrophage tropism and viral persistence: importance of single amino acid changes in the polymerase and glycoprotein of lymphocytic choriomeningitis virus. J Virol 67:7340-7349

Meyer BJ, de la Torre JC, Southern P (2002) Areanviruses: genomic RNAs transcription, and replication. In: Oldstone MB (ed) Arenaviruses. Curr Topics Microbiol Immunol 267:139-158

Oldstone MB (1989) Viral persistence. Cell 56:517-520

Oldstone MB (1991) Molecular anatomy of viral persistence. J Virol 65:6381-6386

Oldstone MB (1998) Viral persistence: mechanisms and consequences. Curr Opin Microbiol 1:436-441

Oldstone MB (2002) Biology and pathogenesis of lymphocytic choriomeningitis virus infection. In: Oldstone MB (ed) Arenaviruses. Curr Topics Microbiol Immunol 267:83-118

Oldstone MB, Rodriguez M, Daughaday WH, Lampert PW (1984) Viral perturbation of endocrine function: disordered cell function leads to disturbed homeostasis and disease. Nature 307:278-281

Oldstone MB, Sinha YN, Blount P, Tishon A, Rodriguez M, von Wedel R, Lampert PW (1982) Virus-induced alterations in homeostasis: alteration in differentiated functions of infected cells in vivo. Science 218:1125-1127

Oldstone MB, Ahmed R, Buchmeier MJ, Blount P, Tishon A (1985) Perturbation of differentiated functions during viral infection in vivo. Relationship of lymphocytic choriomeningitis virus and host strains to growth hormone deficiency. Virology 142:158-174

Pawlotsky JM, Germanidis G, Neumann AU, Pellerin M, Frainais PO, Dhumeaux D (1998) Interferon resistance of hepatitis $C$ virus genotype $1 \mathrm{~b}$ : relationship to nonstructural 5A gene quasispecies mutations. J Virol 72:2795-2805

Perez M, Craven RC, de la Torre JC (2003) The small RING finger protein Z drives arenavirus budding: implications for antiviral strategies. Proc Natl Acad Sci U S A 100:12978-12983

Pinschewer DD, Perez M, Sanchez AB, de la Torre JC (2003) Recombinant lymphocytic choriomeningitis virus expressing vesicular stomatitis virus glycoprotein. Proc Natl Acad Sci U S A 100:7895-7900 
Pircher H, Moskophidis D, Rohrer U, Burki K, Hengartner H, Zinkernagel RM (1990) Viral escape by selection of cytotoxic T cell-resistant virus variants in vivo. Nature 346:629-633

Riviere Y (1987) Mapping arenavirus genes causing virulence. Curr Top Microbiol Immunol 133:59-65

Riviere Y, Oldstone MB (1986) Genetic reassortants of lymphocytic choriomeningitis virus: unexpected disease and mechanism of pathogenesis. J Virol 59:363-368

Riviere Y, Ahmed R, Southern P, Oldstone MB (1985a) Perturbation of differentiated functions during viral infection in vivo. II. Viral reassortants map growth hormone defect to the SRNA of the lymphocytic choriomeningitis virus genome. Virology 142:175-182

Riviere Y, Ahmed R, Southern PJ, Buchmeier MJ, Oldstone MB (1985b) Genetic mapping of lymphocytic choriomeningitis virus pathogenicity: virulence in guinea pigs is associated with the LRNA segment. J Virol 55:704-709

Riviere Y, Ahmed R, Oldstone MB (1986) The use of lymphocytic choriomeningitis virus reassortants to map viral genes causing virulence. Med Microbiol Immunol (Berl) 175:191-192

Ruiz-Jarabo CM, Ly C, Domingo E, de la Torre JC (2003) Lethal mutagenesis of the prototypic arenavirus lymphocytic choriomeningitis virus (LCMV) Virology 308:37-47

Salvato MS (1993) Molecular biology of the prototype arenavirus, lymphocytic choriomeningitis virus. In: Salvato MS (ed) The arenaviridae. Plenum, New York, pp 133-156

Salvato MS, Shimomaye EM (1989) The completed sequence of lymphocytic choriomeningitis virus reveals a unique RNA structure and a gene for a zinc finger protein. Virology 173:1-10

Salvato M, Shimomaye EM, Southern P, Oldstone MBA (1988) Virus-lymphocyte interactions: IV. Molecular characterization of LCMV Armstrong (CTL+) small genomic segment and that of its variant, clone $13\left(\mathrm{CTL}^{-}\right)$. Virology 164:517-522

Salvato M, Shimomaye E, Oldstone MB (1989) The primary structure of the lymphocytic choriomeningitis virus L gene encodes a putative RNA polymerase. Virology 169:377-384

Salvato M, Borrow P, Shimomaye E, Oldstone MB (1991) Molecular basis of viral persistence: a single amino acid change in the glycoprotein of lymphocytic choriomeningitis virus is associated with suppression of the antiviral cytotoxic Tlymphocyte response and establishment of persistence. J Virol 65:1863-1869

Salvato MS, Schweighofer KJ, Burns J, Shimomaye EM (1992) Biochemical and immunological evidence that the $11 \mathrm{kDa}$ zinc-binding protein of lymphocytic choriomeningitis virus is a structural component of the virus. Virus Res 22:185-198

Sevilla N, Kunz S, Holz A, Lewicki H, Homann D, Yamada H, Campbell KP, de La Torre JC, Oldstone MB (2000) Immunosuppression and resultant viral persistence by specific viral targeting of dendritic cells. J Exp Med 192:1249-1260

Sevilla N, Domingo E, de la Torre JC (2002) Contribution of LCMV towards deciphering biology of quasispecies in vivo. Curr Top Microbiol Immunol 263:197-220

Sevilla N, McGavern DB, Teng C, Kunz S, Oldstone MB (2004) Viral targeting of hematopoietic progenitors and inhibition of DC maturation as a dual strategy for immune subversion. J Clin Invest 113:737-745 
Southern PJ (1996) Arenaviridae: the virus and their replication. In: Fields BN, Knipe DM, Howley PM (eds) Fields virology, 3rd edn. Lippincott-Raven, Philadelphia, pp 1505-1519

Teng MN, Borrow P, Oldstone MB, de la Torre JC (1996a) A single amino acid change in the glycoprotein of lymphocytic choriomeningitis virus is associated with the ability to cause growth hormone deficiency syndrome. J Virol 70:8438-8443

Teng MN, Oldstone MB, de la Torre JC (1996b) Suppression of lymphocytic choriomeningitis virus-induced growth hormone deficiency syndrome by diseasenegative virus variants. Virology 223:113-119

Tishon A, Oldstone MB (1990) Perturbation of differentiated functions during viral infection in vivo. In vivo relationship of host genes and lymphocytic choriomeningitis virus to growth hormone deficiency. Am J Pathol 137:965-969

Valsamakis A, Riviere Y, Oldstone MB (1987) Perturbation of differentiated functions in vivo during persistent viral infection. III. Decreased growth hormone mRNA. Virology 156:214-220

Weaver SC, Salas RA, de Manzione N, Fulhorst CF, Duno G, Utrera A, Mills JN, Ksiazek TG, Tovar D, Tesh RB (2000) Guanarito virus (Arenaviridae) isolates from endemic and outlying localities in Venezuela: sequence comparisons among and within strains isolated from Venezuelan hemorrhagic fever patients and rodents. Virology 266:189-195

Weaver SC, Salas RA, de Manzione N, Fulhorst CF, Travasos da Rosa AP, Duno G, Utrera A, Mills JN, Ksiazek TG, Tovar D, Guzman H, Kang W, Tesh RB (2001) Extreme genetic diversity among Pirital virus (Arenaviridae) isolates from western Venezuela. Virology 285:110-118

Welsh RM, Pfau CJ (1972) Determinants of lymphocytic choriomeningitis interference. J Gen Virol 14:177-187 